\title{
Tirofiban facilitates the reperfusion process during endovascular thrombectomy in ICAS
}

\author{
HONGCHEN ZHAO ${ }^{1}$, JINHUA ZHANG ${ }^{1}$, DANYAN GU², ZONGJIE SHI ${ }^{1}$, \\ JIE PAN $^{1}$, YU GENG $^{1}$ and TIANMING SHI ${ }^{1}$ \\ ${ }^{1}$ Department of Neurology, Zhejiang Provincial People's Hospital, Hangzhou, Zhejiang 310014; \\ ${ }^{2}$ Department of Intensive Care Medicine, Xiasha Hospital, Hangzhou, Zhejiang 310018, P.R. China
}

Received February 3, 2017; Accepted July 20, 2017

DOI: $10.3892 /$ etm.2017.4856

\begin{abstract}
The aim of the present study was to assess the use of tirofiban injections for rescue therapy following artery reocclusion due to intra-luminal thrombosis during endovascular thrombectomy in patients with acute ischemic stroke (AIS). A total of seven cases of patients treated with adjunctive tirofiban injections following failed endovascular thrombectomy due to instant intra-luminal thrombosis were retrospectively assessed. A Solitaire stent was used as the primary thrombectomy device in all patients. Tirofiban was injected intra-arterially via a temporarily deployed Solitaire stent with continuous intravenous infusion for the subsequent $24 \mathrm{~h}$; half of the conventionally recommended dose was employed. Outcome measures included angiographic reperfusion (mTICI), symptomatic intracranial hemorrhage, mortality and functional independence at 90 days (modified Rankin Scale, 0-2). Six patients had occlusions in the middle cerebral artery and one patient had occlusions in the basilar artery. Of the seven patients, five exhibited successful reperfusion (mTICI 2b-3) and achieved functional independence following 90 days. Reperfusion failed in the remaining two patients, who succumbed within 90 days of therapy. No intracranial or extracranial hemorrhage cases were identified. The results of the present study suggest that tirofiban facilitates reperfusion and ameliorates long-term prognosis in patients with AIS undergoing endovascular thrombectomy, and may be safe for those receiving intravenous tissue plasminogen activator therapy.
\end{abstract}

\section{Introduction}

In 2015, five randomized trials showed improved efficacy of endovascular thrombectomy compared with standard

Correspondence to: Dr Tianming Shi, Department of Neurology, Zhejiang Provincial People's Hospital, 158 Shangtang Road, Hangzhou, Zhejiang 310014, P.R. China

E-mail: shitm2005@163.com

Key words: acute ischemic stroke, endovascular thrombectomy, tirofiban, intra-luminal thrombosis medical care in patients with acute ischemic stroke (AIS) caused by arterial occlusion in proximal anterior circulation (1-5). This procedure is considered the standard of care for patients with proximal anterior circulation occlusions (6). However, the reperfusion rate ranges from 58.7 to $88 \%$ (1-5), indicating that some patients still display failed reperfusion. Compared with the patients included in the previous 5 trials, more individuals with AIS have intracranial atherosclerosis stenosis (ICAS) (7-10) in Asia. However, ICAS related arterial occlusion is associated with longer procedure time and poorer clinical outcome (11-13). Further rate increase of successful and complete reperfusion is needed. Several methods, including balloon angioplasty, stent angioplasty (14) and intra-arterial injection of alteplase, have been used as rescue therapies. However, drawbacks, such as further injury of vessel intima and increased intracranial hemorrhage, limit their applications. As different mechanisms are considered, we evaluated some adjunctive methods listed above to facilitate reperfusion. Tirofiban, a glycoprotein IIb/IIIa antagonist, can block fibrin-binding receptors reversibly, preventing platelet aggregation and subsequent thrombosis effectively. Since hyperacute thrombosis intra-vessel/intra-stent is considered the reason for reperfusion failure, we injected tirofiban intra-arterially through the deployed Solitaire stent, and pumped intravenously thereafter. Here, we assessed the efficacy and safety of this adjunctive protocol in our center.

\section{Materials and methods}

Patients. A total of 246 consecutive AIS patients with onset within $6 \mathrm{~h}$ were identified from our stroke database from March 2015 to April 2016.

Inclusion criteria. i) Onset within $6 \mathrm{~h}$ and meeting the criteria for thrombectomy (6). ii) Persistent occlusion despite initial endovascular thrombectomy assessed by digital subtraction angiography (DSA). iii) Informed consent signed by any authorized person.

Procedure. All patients underwent cerebral CT/CTA upon arrival at our hospital. Concurring with the indications for intra-venous thrombolysis, t-PA was injected intravenously first, and endovascular therapy was initiated after spotting the 

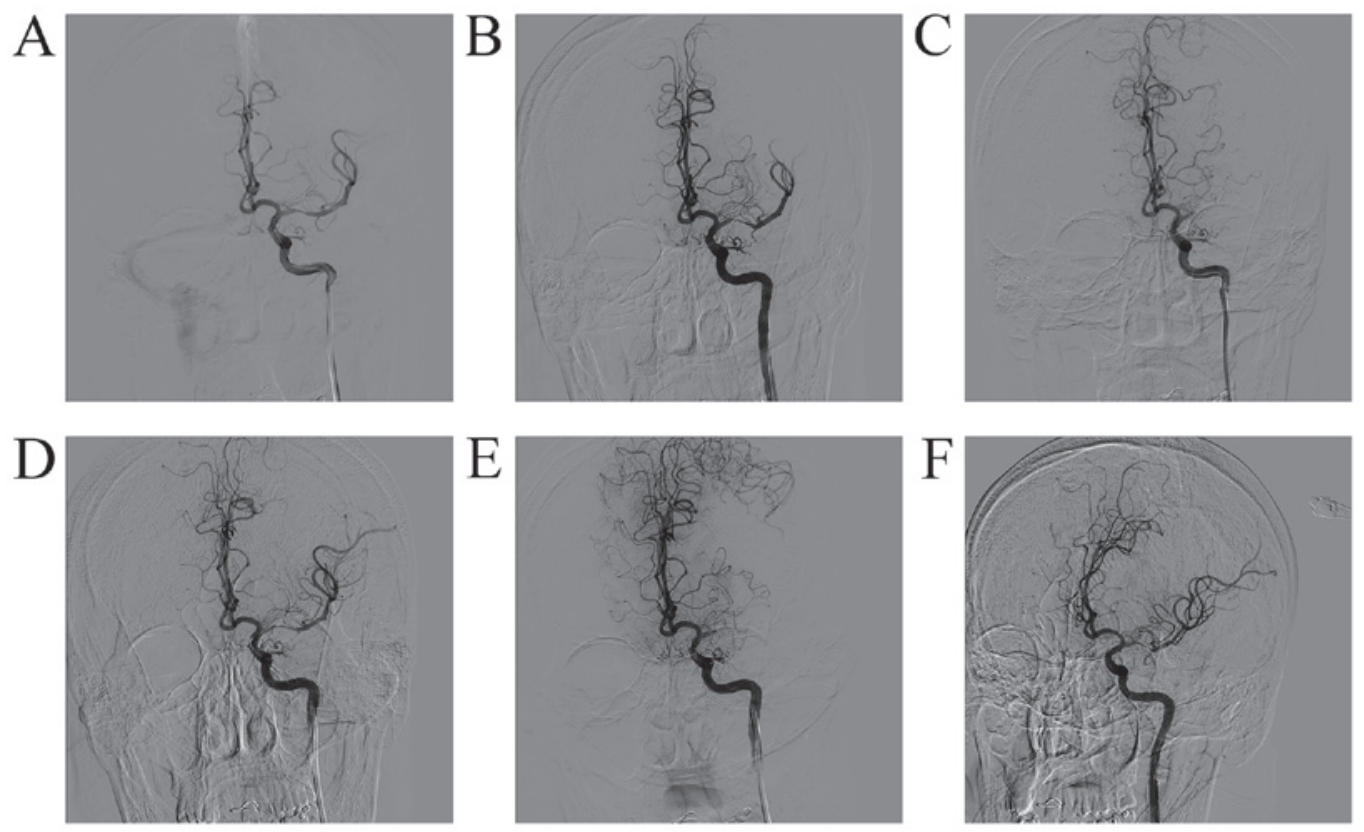

Figure 1. (A) First deployment of solitaire AB; (B) severe stenosis after stent retrieval; (C) artery reocclusion 5 min after stent retrieval; (D) secondary deployment of solitaire $\mathrm{AB}$; (E) artery reocclusion due to intraluminal (intra-stent) thrombosis; (F) tirofiban adjunctive reperfusion.

intracranial large artery occlusion. Endovascular thrombectomy was performed under local anesthesia with lidocaine plus conscious sedation with dexmedetomidine. A guiding catheter continuously flushed with heparinized saline was placed in the intrapetrous carotid artery. A microcatheter with a 0.014 inch micro-guidewire was navigated distally to the thrombus. Then, the Solitaire stent was deployed across the clot for 5 min before retrieval, during which proximal aspiration with a $50 \mathrm{cc}$ syringe was performed through the guiding catheter. Throughout the process of endovascular thrombectomy, cerebral angiography was performed to assess reperfusion immediately after stent deployment and $5 \mathrm{~min}$ later. When reocclusion occurred before stent retrieval, intra-stent thrombosis was suspected. In this case, we first attempted to retrieve the stent, and angiography followed immediately; 15 min later, failure to obtain stable reperfusion implied redeployment of the stent combined with tirofiban as remedy. Adjuvant tirofiban was used at $0.05 \mathrm{mg} / \mathrm{min}$ through the guiding catheter intra-arterially for $5 \mathrm{~min}$, and a loading dose of $0.2 \mu \mathrm{g} / \mathrm{kg} / \mathrm{min}$ administered intravenously for $30 \mathrm{~min}$; a maintenance dose of $0.05 \mu \mathrm{g} / \mathrm{kg} / \mathrm{min}$ was pumped intravenously thereafter for $24 \mathrm{~h}$. Then, the Solitaire stent and delivery microcatheter were withdrawn after injection of the loading dose of tirofiban. A typical procedure is presented in Fig. 1. In case of unsuccessful reperfusion, Solitaire thrombectomy was repeated according to the operator's discretion, for a maximum of three attempts.

Data collection. Patient characteristics were obtained from medical records: Age, gender, NIHSS, treatment with intravenous thrombolysis, time between symptom onset to groin puncture, and stroke etiology catalogued by TOAST. Images were reviewed for arterial occlusive lesion (AOL) site and reperfusion grade according to the modified Thrombolysis in Cerebral Infarction score (mTICI): grade 0 , no perfusion; grade 1, perfusion beyond the initial obstruction but limited distal branch filling with little or slow distal perfusion; grade $2 \mathrm{a}$, perfusion of less than half of the vascular distribution of the occluded artery; grade $2 b$, perfusion of half or more of the vascular distribution of the occluded artery; grade 3 , full perfusion with filling of all distal branches. Symptomatic intracerebral hemorrhage was recorded according to SITS-MOST: type 2 parenchymatous intracerebral hemorrhage with neurological deterioration, defined as an increase of 4 or more points in the NIHSS score (15).

Outcome measures and follow-up. Modified Rankin Scale (mRS) scores were obtained at 90 days; mRS of $0-2$ was considered to reflect a good functional outcome as the primary outcome.

Target vessel reperfusion was assessed using cerebral angiography, and classified by the mTICI score. An mTICI grade $2 b$ or 3 was considered a successful reperfusion as the secondary outcome.

Neurological improvement was assessed via NIHSS scores at $24 \mathrm{~h}$, and defined as a reduction in the NIHSS score of $>4$ points. All patients underwent CT and CTA within $24 \mathrm{~h}$ after the procedure to evaluate hemorrhagic complications and vessel morphology. Post-procedural hemorrhage was assessed as the main complication associated with endovascular thrombectomy.

Safety outcome measures were presence of symptomatic intracranial and extracranial hemorrhages following the procedure, and mortality at 90 days.

\section{Results}

A total of 174/246 patients received t-PA thrombolysis intravenously, and 63 underwent endovascular thrombectomy for large artery occlusion. Among them, 7 patients met the criteria for adjunctive tirofiban injection during endovascular thrombectomy. 


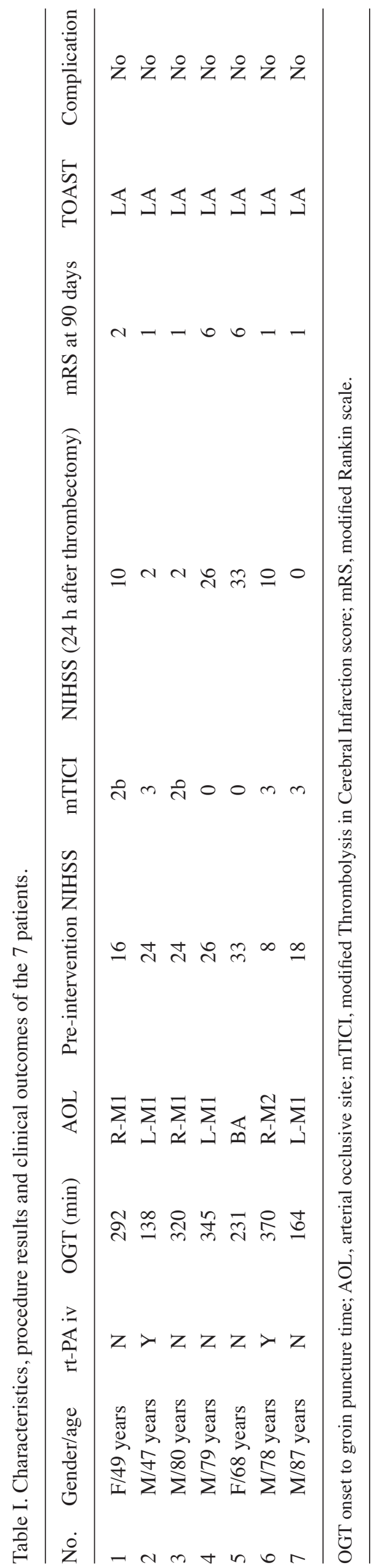

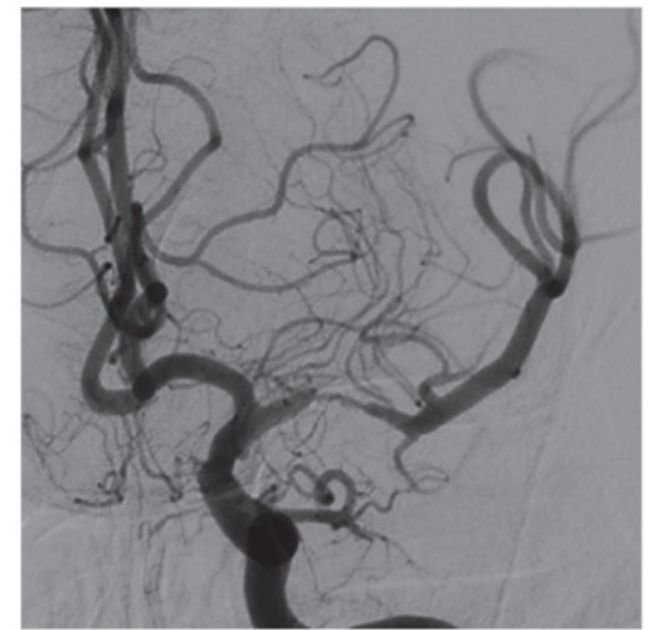

Figure 2. Sign of intraluminal thrombosis-a fluffy filling defect.

Intra-luminal thrombosis was defined as a fluffy filling defect (Fig. 2) in the region of reocclusion after temporary reperfusion following retrieval of the deployed stent. We assessed patients treated with tirofiban injection through the deployed solitaire stent after failed endovascular thrombectomy ascribed to intra-luminal thrombosis.

Baseline characteristics, procedures, and clinical outcomes are shown in Table I. All the patients had large atherosclerosis (LA) as TOAST subtype. Age ranged from 47 to 87 years, with initial NIHSS score ranging between 8 and 26. Time between symptom onset and groin puncture ranged from 138 to $370 \mathrm{~min}$. A total of 2 patients were treated with IV t-PA before endovascular thrombectomy. Solitaire stent was used as first line device for endovascular thrombectomy. All patients were treated with an equal dose of tirofiban based on weight.

Successful reperfusion was achieved in 5 of the 7 patients after tirofiban injection and subsequent Solitaire thrombectomy, and no reocclusion occurred as a consequence of intravenous injection of tirofiban. No patient had intracranial hemorrhage.

Neurological improvement (decrease of the NIHSS score by more than 4 points) at $24 \mathrm{~h}$ and good functional outcome $(\mathrm{mRS} \leq 2)$ at 90 days were observed in patients who completed reperfusion. The two fatalities observed were caused by malignant edema following large cerebral infarction due to unsuccessful reperfusion of middle cerebral artery, despite the use of tirofiban.

\section{Discussion}

Endovascular thrombectomy using a stent retriever is considered an efficient method for blood flow restoration in patients with ischemic stroke due to large intracranial vessel occlusion (1-5). However, failure of reperfusion persists in a subset of patients due to various factors, such as ICAS (11-13). Several techniques for the treatment of cases refractory to endovascular thrombectomy using stent retriever have been previously introduced. Balloon angioplasty is an effective procedure when reperfusion fails with Solitaire stent alone. In a study by Kurre et al (16), other rescue methods were applied, including the use of different stent retrievers, balloon angioplasty, permanent stent implantation, distal aspiration without a retriever, 
and intra-arterial fibrinolysis. Klisch et al (17) recently reported successful reperfusion and good clinical outcome, respectively, in 80 and $50 \%$ of patients administered rescue treatment with the double Solitaire stent retriever technique. However, angioplasty is not considered a method for ICAS management $(18,19)$. Specifically, when perforators exist near the stenotic lesion (e.g., anterior inferior cerebellar arteries in the BA trunk or striatocapsular arteries in the MCA trunk), angioplasty and intracranial stenting should be performed with caution because of frequent perforator occlusions (20). Arterial reocclusion in patients with acute ischemic stroke receiving endovascular intervention occurs at a rate of $18 \%$, and is also an independent predictor of poor long-term outcome (21). In the case of intracranial atherosclerosis stenosis, intraluminal thrombosis is a major cause of reocclusion (13). In multiple studies $(13,22-24)$, the glycoprotein IIb/IIIa antagonist tirofiban has been preliminary used as salvage therapy in patients with unsuccessful reperfusion. In these retrospective studies, tirofiban displayed adjuvant benefits in reperfusion, with relatively low risk of intracranial hemorrhage, and about $50 \%$ patients achieved favorable outcomes thanks to adjuvant tirofiban infusion. Moreover, Li et al (25) had demonstrated the safety and efficacy in a prospective study.

Nonetheless, Kellert et al (26) cautioned about the risk of fatal intracerebral hemorrhage and poor outcome associated with tirofiban in endovascular stroke therapy. In this observational study, tirofiban was not only administered after stent implantation, but also to patients with suspected endothelial damage (for example, after repeated reperfusion attempts). Therefore, it was often at the discretion of the interventionist to administer tirofiban or not, which in turn led to bias.

Based on the above, our center limits the use of tirofiban in intraluminal thrombosis performed during endovascular mechanical thrombectomy in ICAS (intracranial artherosclerosis stenosis), but not in cardioembolism. However, intraluminal thrombosis is always accompanied with severe stenosis in the cerebral artery (27). We distinguish intraluminal thrombosis from stenosis occlusion by the presence of reocclusion immediately after partial reperfusion. In these patients, tirofiban showed efficiency in reperfusion as well as favorable outcomes and safety, in both intra-cerebral and total hemorrhagic complications.

In this retrospective study, $2 / 7$ patients still failed to achieve recanalization; therefore, additional measures should be taken based on the risk to benefit ratio.

Obviously, this study had limitations: small sample size, a retrospective design, and a non-contrasted nature; further investigations are warranted to verify the efficiency and safety of tirofiban adjuvant reperfusion in vessel reocclusion due to intra-luminal thrombosis during endovascular thrombectomy.

In conclusion, injection of tirofiban in cases of intra-luminal thrombosis may facilitate reperfusion and favor neurological outcomes in patients with acute ischemic stroke, and may be safe in combination with t-PA and endovascular thrombectomy.

\section{Refces}

1. BerkhemerOA,FransenPS,BeumerD,vandenBergLA,LingsmaHF, Yoo AJ, Schonewille WJ, Vos JA, Nederkoorn PJ, Wermer MJ, et al; MR CLEAN Investigators: A randomized trial of intraarterial treatment for acute ischemic stroke. N Engl J Med 372: 11-20, 2015.
2. Saver JL, Goyal M, Bonafe A, Diener HC, Levy EI, Pereira VM, Albers GW, Cognard C, Cohen DJ, Hacke W, et al; SWIFT-PRIME Investigators: Stent-retriever thrombectomy after intravenous t-PA vs. t-PA alone in stroke. N Engl J Med 372: 2285-2295, 2015.

3. Jovin TG, Chamorro A, Cobo E, de Miquel MA, Molina CA Rovira A, San Román L, Serena J, Abilleira S, Ribó M, et al; REVASCAT Trial Investigators: Thrombectomy within $8 \mathrm{~h}$ after symptom onset in ischemic stroke. N Engl J Med 372: 2296-2306, 2015.

4. Goyal M, Demchuk AM, Menon BK, Eesa M, Rempel JL, Thornton J, Roy D, Jovin TG, Willinsky RA, Sapkota BL, et al; ESCAPE Trial Investigators: Randomized assessment of rapid endovascular treatment of ischemic stroke. N Engl J Med 372: 1019-1030, 2015.

5. Campbell BC, Mitchell PJ, Kleinig TJ, Dewey HM, Churilov L, Yassi N, Yan B, Dowling RJ, Parsons MW, Oxley TJ, et al; EXTEND-IA Investigators: Endovascular therapy for ischemic stroke with perfusion-imaging selection. N Engl J Med 372: 1009-1018, 2015.

6. Powers WJ, Derdeyn CP, Biller J, Coffey CS, Hoh BL, Jauch EC, Johnston KC, Johnston SC, Khalessi AA, Kidwell CS, et al; American Heart Association Stroke Council: 2015 American Heart Association/American Stroke Association focused update of the 2013 guidelines for the early management of patients with acute ischemic stroke regarding endovascular treatment: A guideline for healthcare professionals from the American Heart Association/American Stroke Association. Stroke 46: 3020-3035, 2015

7. Wang Y, Zhao X, Liu L, Soo YO, Pu Y, Pan Y, Wang Y, Zou X, Leung TW, Cai Y, et al; CICAS Study Group: Prevalence and outcomes of symptomatic intracranial large artery stenoses and occlusions in China: The Chinese Intracranial Atherosclerosis (CICAS) Study. Stroke 45: 663-669, 2014.

8. De Silva DA, Woon FP, Lee MP, Chen CP, Chang HM and Wong MC: South Asian patients with ischemic stroke: Intracranial large arteries are the predominant site of disease. Stroke 38: 2592-2594, 2007.

9. Lee SJ, Cho SJ, Moon HS, Shon YM, Lee KH, Kim DI, Lee BB, Byun HS, Han SH and Chung CS: Combined extracranial and intracranial atherosclerosis in Korean patients. Arch Neurol 60: 1561-1564, 2003.

10. Suwanwela NC and Chutinetr A: Risk factors for atherosclerosis of cervicocerebral arteries: Intracranial versus extracranial. Neuroepidemiology 22: 37-40, 2003.

11. Kim YW, Hong JM, Park DG, Choi JW, Kang DH, Kim YS, Zaidat OO, Demchuk AM, Hwang YH and Lee JS: Effect of intracranial atherosclerotic disease on endovascular treatment for patients with acute vertebrobasilar occlusion. AJNR Am J Neuroradio: Jun 16, 2016 (Epub ahead of print).

12. Lee JS, Hong JM, Lee KS, Suh HI, Choi JW and Kim SY: Primary stent retrieval for acute intracranial large artery occlusion due to atherosclerotic disease. J Stroke 18: 96-101, 2016.

13. Kang DH, Kim YW, Hwang YH, Park SP, Kim YS and Baik SK: Instant reocclusion following mechanical thrombectomy of in situ thromboocclusion and the role of low-dose intra-arterial tirofiban. Cerebrovasc Dis 37: 350-355, 2014.

14. Yoon W, Kim SK, Park MS, Kim BC and Kang HK: Endovascular treatment and the outcomes of atherosclerotic intracranial stenosis in patients with hyperacute stroke. Neurosurgery 76: 680-686, 2015.

15. Wahlgren N, Ahmed N, Dávalos A, Ford GA, Grond M, Hacke W, Hennerici MG, Kaste M, Kuelkens S, Larrue V, et al; SITS-MOST Investigators: Thrombolysis with alteplase for acute ischaemic stroke in the safe implementation of thrombolysis in stroke-monitoring study (SITS-MOST): An observational study. Lancet 369: 275-282, 2007.

16. Kurre W, Aguilar-Pérez M, Schmid E, Sperber W, Bäzner H and Henkes H: Clinical experience with the pREset stent retriever for the treatment of acute ischemic stroke - a review of 271 consecutive cases. Neuroradiology 56: 397-403, 2014.

17. Klisch J, Sychra V, Strasilla C, Taschner CA, Reinhard M, Urbach $\mathrm{H}$ and Meckel S. Double solitaire mechanical thrombectomy in acute stroke: Effective rescue strategy for refractory artery occlusions? AJNR Am J Neuroradiol 36: 552-556, 2015.

18. Zaidat OO, Fitzsimmons BF, Woodward BK, Wang Z, Killer-Oberpfalzer M, Wakhloo A, Gupta R, Kirshner H, Megerian JT, Lesko J, et al; VISSIT Trial Investigators: Effect of a balloon-expandable intracranial stent vs medical therapy on risk of stroke in patients with symptomatic intracranial stenosis: The VISSIT randomized clinical trial. JAMA 313: 1240-1248, 2015. 
19. Chimowitz MI, Lynn MJ, Derdeyn CP, Turan TN, Fiorella D, Lane BF, Janis LS, Lutsep HL, Barnwell SL, Waters MF, et al; SAMPRISS Trial Investigators: Stenting versus aggressive medical therapy for intracranial arterial stenosis. N Engl J Med 365: 993-1003, 2011.

20. Fiorella D, Derdeyn CP, Lynn MJ, Barnwell SL, Hoh BL, Levy EI, Harrigan MR, Klucznik RP, McDougall CG, Pride GL Jr, et al; SAMPRISS Trial Investigators: Detailed analysis of periprocedural strokes in patients undergoing intracranial stenting in stenting and aggressive medical management for preventing recurrent stroke in intracranial stenosis (SAMMPRIS). Stroke 43: 2682-2688, 2012.

21. Janjua N, Alkawi A, Suri MF and Qureshi AI: Impact of arterial reocclusion and distal fragmentation during thrombolysis among patients with acute ischemic stroke. AJNR Am J Neuroradiol 29: 253-258, 2008

22. Seo JH, Jeong HW, Kim ST and Kim EG: Adjuvant tirofiban injection through deployed solitaire stent as a rescue technique after failed mechanical thrombectomy in acute stroke. Neurointervention 10: 22-27, 2015.
23. Kim JW, Jeon P, Kim GM, Bang OY, Byun HS and Kim KH: Local intraarterial tirofiban after formation of anterograde flow in patients with acute ischemic stroke: Preliminary experience and short term follow-up results. Clin Neurol Neurosurg 114: 1316-1319, 2012.

24. Kwon JH, Shin SH, Weon YC, Hwang JC and Baik SK Intra-arterial adjuvant tirofiban after unsuccessful intra-arterial thrombolysis of acute ischemic stroke: Preliminary experience in 16 patients. Neuroradiology 53: 779-785, 2011.

25. Li W, Lin, Lu Zhang M, Wu Y, Liu C, Li X, Huang S, Liang C, Wang Y, Chen J and Feng W: Safety and preliminary efficacy of early tirofiban treatment after alteplase in acute ischemic stroke patients. Stroke 47: 2649-2651, 2016.

26. Kellert L, Hametner C, Rohde S, Bendszus M, Hacke W, Ringleb P and Stampfl S: Endovascular stroke therapy: Tirofiban is associated with risk of fatal intracerebral hemorrhage and poor outcome. Stroke 44: 1453-1455, 2013.

27. Buchan A, Gates P, Pelz D and Barnett HJ: Intraluminal thrombus in the cerebral circulation. Implications for surgical management. Stroke 19: 681-687, 1988. 